\title{
Target Detection in Hyperspectral Imagery Combining Spectral and Spatial Features
}

\author{
Serkan Salturk ${ }^{1}$, Abdullah $\mathrm{Bal}^{2}$, and Hamidullah Binol ${ }^{2}$
}

\begin{abstract}
Hyperspectral images are high dimensional data compared to RGB and gray level images. As well as these images have lots of decisive feature in spectral domain, they have plenty of information about the target also in spatial domain. Hence, performance of target detection algorithms can be improved when spatial and spectral features are employed together. In this paper, Covariance Descriptor method which enable combining of spectral and spatial features are used for target detection. In addition, Kernel Covariance Descriptor technique is developed utilizing kernel approach. Efficient spectral and spatial features are determined by experimental studies. The target detection performance is compared with each other. The experiments on two real hyperspectral data sets demonstrate that the proposed scheme is effective for target detection problems.
\end{abstract}

Keywords - Covariance descriptor; hyperspectral imaging; kernel methods; spatial features, spectral features.

\section{INTRODUCTION}

Due to rapid development of hyperspectral imaging technology, working areas like target detection, classification on hyperspectral imagery become more popular. The use of hyperspectral imagery systems on military as target recognition, mine, chemical and radioactive attack detection, on agriculture as accurate and economical pesticide, qualified production, also on medical, mining, food areas are often encountered [1-6]. These systems, depending on their multiband structure, contain much more information about the target than the conventional images. As the number of bands increase, the system will be more sensitive.

Each pixel on hyperspectral images is represented with a vector which is obtained from values of bands according to hyperspectral sensor characteristics. The processes such as target detection, classification, recognition in hyperspectral images are performed comparing with these vectors. The vector which represents a specific material is called spectral signature. The spectral signatures of soil, human body, river or lake are different from each other.

While working on hyperspectral imagery, spectral features are generally utilized. Besides, using spatial features that comprise a great deal of information about the data may affect the success rate positively. In this paper, we have applied Covariance Descriptor (CD) method combining the spectral and spatial features and also Kernel Covariance Descriptor $(\mathrm{KCD})$ is employed in hyperspectral images.

${ }^{1}$ Department of Informatics, ${ }^{2}$ Department of Electronics and Communication Engineering, Yildiz Technical University, 34220 Istanbul, Turkey

\section{METHODS}

\section{A. Covariance Descriptor (CD)}

Classic $\mathrm{CD}$ is based on representing region (the target region or the whole image) using the features of the pixels of the region in spatial domain. On hyperspectral imagery the features are obtained from the spectral vectors to create feature and covariance matrix which is used for the comparison of classes [7]. One of the main advantages of this method is the independence of the covariance matrix from the size of spectral vector [8]. It is no matter how long the vector is, the number of features determines size of the covariance matrix. In Eq. (1) the feature matrix formed by utilized features for one pixel of a spectral vector is shown.

$$
\emptyset(x)=\left[x_{v} I(x), I_{x x} I_{x x s} \sqrt{I_{x}^{2}+I_{x x}^{2}}\right]
$$

where $I$ is for spectral vector, $x$ for band number of vector, $I(x)$ for reflectance value, $I_{x}$ and $I_{x x}$ are for first and second derivatives, respectively. After getting all of these feature vectors for all of the pixels of vector $5 \times 5$ covariance matrix is constructed.

As the covariance matrices are positive semidefinite, Euclidean distance cannot be used to compare two classes. To evaluate these matrices, the Eq. (2) is utilized [9].

$$
\rho\left(C_{1}, C_{2}\right)=\sqrt{\sum_{i=1}^{n} l^{2} \lambda_{i}\left(C_{1}, C_{2}\right)}
$$

Here $C_{1}$ and $C_{2}$ are covariance matrices and $\lambda_{i}\left(C_{1}, C_{2}\right)$ refers to eigenvectors of these generalized matrices.

\section{B. Kernel Covariance Descriptor (KCD)}

Although classical CD returns successful results on several datasets, more efficient methods are needed to work on some of classes on hyperspectral datasets. Hence, Kernel Covariance Descriptor (KCD) that changes the distribution of the data by mapping the feature vectors from classic $\mathrm{CD}$ by kernel trick is proposed in [10-11]. By this method, the separability of classes and target detection performance are improved.

In this application, Gaussian kernel is applied but the normalization constant is omitted. Such kind of function is called Radial Basis Function (RBF):

$$
K\left(x_{i}, x_{j}\right)=\exp \left(\frac{-\left\|x_{i}-x_{j}\right\|}{\sigma^{2}}\right)
$$

While creating kernel covariance matrix, the feature vectors found for every pixel of spectral vector are compared with kernel function and a real value is found for each comparison. 
These values form the kernel covariance matrices and we represent every pixel of dataset by these matrices.

\section{Combination of Spectral and Spatial Features}

Spectral features are generally used in hyperspectral image processing. However, the features on spatial domain have important information about the dataset. Therefore, feature matrix is constructed with the spatial and the spectral features. $\emptyset(x)=\left[x_{x} I(x), I_{x x} I_{x x^{x}} \sqrt{I_{x}{ }^{2}+I_{x x}{ }^{2}}, \operatorname{Im}(x), \operatorname{Im}_{x^{x}} \operatorname{Im} m_{x x} \ldots\right](4)$

In equation (4), $\mathrm{Im}$ stands for spatial mean of $3 \times 3$ matrix on every band and $I m_{x}, I m_{x x}$ stand for first and second derivatives of reflectance values respectively. However, as spatial feature values are similar to spectral feature values, CD and KCD performances decrease. Based on this, the spectral features are used before applying $\mathrm{CD}$ or $\mathrm{KCD}$ and a new vector for target pixel is formed utilizing the pixels on spatial domain.

In this paper, we only used mean feature. A target window size is determined and a new vector is created by calculating mean of the pixels for each band. It is analyzed that the performance of target detection is increased including mean feature.

\section{EXPERIMENTAL RESULTS}

AVIRIS Indian Pine is one of the datasets that we have applied $\mathrm{CD}$ and $\mathrm{KCD}$ algorithms for target detection. It contains 159 bands when the noised and bad bands are discarded. Other dataset CATALCAO1 contains 196 spectral bands.

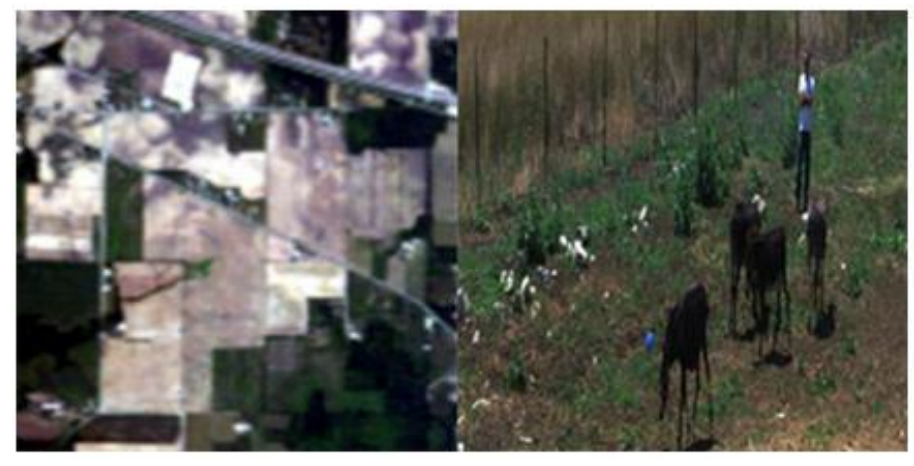

a)

b)

Fig. 1 (a) RGB image for Indian Pine (b) RGB image for CATALCA01

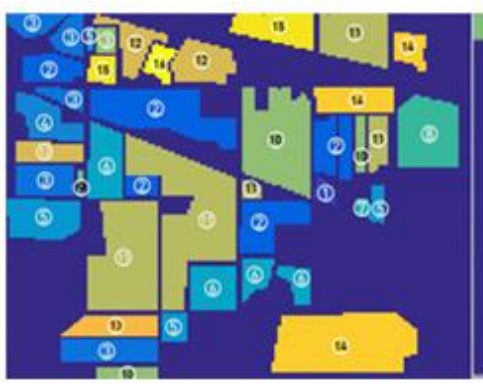

a)

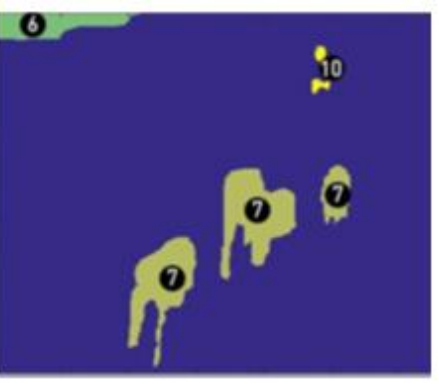

b)
Fig. 2: (a) Ground truth map for Indian Pine (b) Part of ground truth data for CATALCA01
Figure 1(a) and b) shows the RGB images for datasets Indian Pine and CATALCAO1 respectively. Figure 2(a) shows the ground truth data for AVIRIS Indian Pine dataset and Figure 1(b) shows CATALCAO1 dataset including only classes in use. Because of the massiveness of this dataset, only this part of the image is used for application.

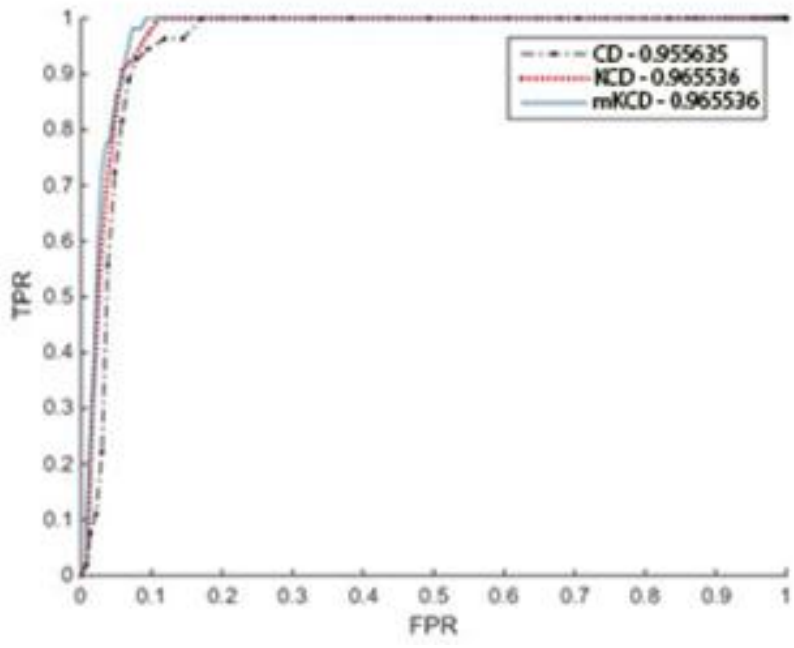

a)

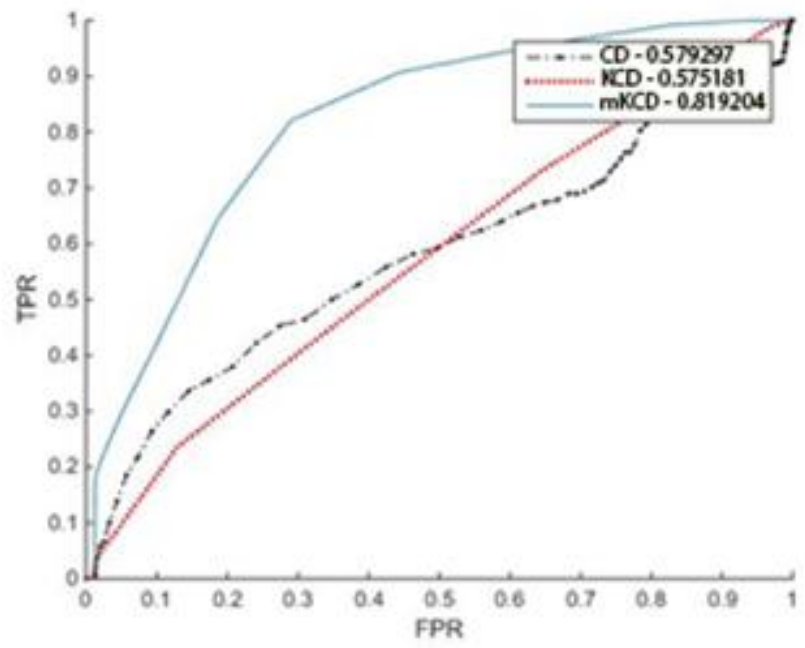

b)

Fig. 2 (a) ROC curves for Indian Pine alfalfa (class 1) class (b) ROC curves for CATALCA01 human (class 10) class

In this paper, before applying $\mathrm{CD}$ and KCD methods, firstly, the spectral and spatial features are combined in the feature vectors. In order to improve the detection efficiency, spatial features are discarded from feature vectors. The results of $\mathrm{CD}$, $\mathrm{KCD}$ and the new mean vector's that is calculated from the spatial mean value on each band KCD (mKCD) are evaluated. First five classes of Indian Pine and two classes of CATALCAO1 are included. The mean vectors are constructed as $3 \times 3$ window size. This window size can vary based on the dataset.

Table I and Table II give the area under the recall-false positive rate curves (AUCs) for Indian Pine and CATALCAO1 datasets, respectively. Test results on AVIRIS Indian Pine and CATALCA01 hyperspectral datasets show that including 
spatial features with spectral features improves the target detection performance. Figure 2(a) and (b) show the results for Indian Pine alfalfa class and CATALCAO1 human class, respectively. For this paper, on spatial domain, only mean feature is employed. KCD is also utilized via a kernel function instead of classical CD. It is analyzed that KCD target detection performance is better than classical $\mathrm{CD}$ for most of the classes. Including spatial features via feature vector returns worse results while the new vector is created with spatial features show up better results. For Indian Pine dataset, first 5 classes, for CATALCAO1 dataset 6 and 10th classes are tested.

TABLE I: AREA UNDER RECALL - FALSE POSITIVE RATE CURVES FOR SELECTED CLASSES OF AVIRIS INDIAN PINE DATASET

\begin{tabular}{llll}
\hline \hline Classes & CD & KCD & mKCD \\
\hline Alfalfa & 0.9487 & 0.9571 & $\underline{0.9602}$ \\
Corn-notill & 0.5856 & 0.6264 & $\underline{0.6391}$ \\
Corn-mintill & 0.6864 & 0.7082 & $\underline{0.7152}$ \\
Corn & 0.6249 & 0.6889 & $\underline{0.6911}$ \\
Grass-pasture & 0.8678 & 0.8688 & $\underline{0.8702}$ \\
\hline \hline
\end{tabular}

TABLE II: AREA UNDER RECALL - FALSE POSITIVE RATE CURVES FOR SELECTED CLASSES OF CATALCA01 DATASET

\begin{tabular}{llll}
\hline \hline Classes & CD & KCD & mKCD \\
\hline Wet heath & 0.9613 & 0.8367 & $\underline{0.9946}$ \\
Human & 0.5882 & 0.5908 & $\underline{0.8018}$ \\
\hline \hline
\end{tabular}

\section{CONCLUSION}

In this paper, Kernel Covariance Descriptor (KCD) is employed instead of Classical Covariance Descriptor (CD) for target detection in hyperspectral imagery. Spatial features are also included in our application. Firstly, the spatial features are joined as the part of feature vectors. However, the target detection performance of these new feature vectors is not satisfactory. Hence, we applied spatial features before constructing the feature vectors to improve the performance of target detection. During the tests AVIRIS Indian Pine and CATALCAO1 datasets are utilized. Because of the massiveness of CATALCAO1 dataset, only a small part of it is applied. The outputs of the target detection application show that KCD method returns better results than $\mathrm{CD}$ and including spatial features improves the success.

\section{ACKNOWLEDGMENT}

This research was supported by a grant from The Scientific and Technological Research Council of Turkey (TUBITAK 112E207). The authors would like to thank the YTU-YAZGI Laboratory Team for providing the CATALCA01 HSI data.

\section{REFERENCES}

[1] J. Goutsias, and A. Banerji, "A morphological approach to automatic mine detection problems", IEEE Trans. Aerospace and Electronic Systems, vol. 34, No. 4, pp.1085-1096, 1998. http://dx.doi.org/10.1109/7.722683

[2] G. Lu and B. Fei, "Medical hyperspectral imaging: A review" Journal of Biomedical Optics, 19(1), 10901. doi:10.1117/1.JBO.19.1.010901, 2014.

[3] Z. Liu, H. Wang and Q. Li, "Tongue tumor detection in medical hyperspectral images", Sensors, 12(1), 162-174. doi:10.3390/s120100162, 2012.
[4] I. Burud, C. Moni, A. Flo, C. Futsaether, M. Steffens, and D. Rasse, "Qualitative and quantitative mapping of biochar in a soil profile using hyperspectral imaging", Soil and Tillage Research, 155, 523-531. doi:10.1016/j.still.2015.06.020, 2015.

[5] H. Binol, G. Bilgin, S. Dinc, and A. Bal, "Kernel Fukunaga-Koontz Transform Subspaces for Classification of Hyperspectral Images with Small Sample Sizes". IEEE Geoscience and Remote Sensing Letters, 12(6), 1287-1291, 2015. http://dx.doi.org/10.1109/LGRS.2015.2393438

[6] H. Binol., Uslu, F. S., and A. Bal, "Unsupervised Nonlinear Feature Extraction Method And Its Effects On Target Detection In HighDimensional Data". International Journal of Electrical, Electronics and Data Communication, 3(8), 43-46, 2015.

[7] H. Binol, A. Bal and S. Dinc, "Classification on hyperspectral images using enhanced covariance descriptor," 20th IEEE Signal Processing and Communication Applications Conference (SIU), 2012. http://dx.doi.org/10.1109/siu.2012.6204685

[8] O. Tuzel, F. Porikli and M. Peter, "Fast Covariance: A fast Descriptor for Detection and Classification", Proc. 9th Europian Conf. On Computer Vision, 589-600, 2006.

[9] W. Förstner, B. Moonen, "A metric for covariance matrices". Technical report, Dept. Of Geodesy and Geoinformatics, Stuttgart University, 1999.

[10] S. Saltürk, "Target Detection on Hyperspectral Images with Composite Kernel Covariance Descriptor," Master Thesis, YTÜ, 2015.

[11] Binol, H., Bal, A., and Cukur, H. (2015, September). Differential evolution algorithm-based kernel parameter selection for FukunagaKoontz Transform Subspaces Construction. The 22nd SPIE Symposium on Remote Sensing. Toulouse, France. 